\title{
Queueing Analysis of Performance Measures under A New Configurable Channel Allocation in Cognitive Radio
}

\author{
Shi Wang Student Member, IEEE, B.T. Maharaj Senior Member, IEEE, Attahiru S. Alfa Member, IEEE
}

\begin{abstract}
To help achieve optimal channel allocation for a cognitive radio network with multiple channels and multiple users, the authors propose an queueing analytical framework for implementing a configurable channel allocation method. Various allocation scenarios can be captured by the allocation method under different parameters. The influence of the parameters under different environmental parameters and system settings on the performance measures is investigated by the proposed framework. System settings such as imperfect spectrum sensing, adaptive modulation and coding scheme, automatic repeat and request, various primary user activity patterns and finite secondary user buffer size are considered to make the proposed model practical. The procedures to derive performance measures, such as average queue length, packet loss rate, throughput and average packet delay, are presented. Numerical results that reveal the relationship between various performance measures and the channel allocation method parameters under different channel conditions and secondary user system settings are shown. The allocation method and the analytical framework would be useful to help achieve optimal performance and evaluate different allocation methods.
\end{abstract}

Index Terms-Cognitive radio, channel allocation method, configurable, queueing analysis, performance evaluation.

\section{INTRODUCTION}

With the growth in the demand for speed and quality of telecommunication, the radio spectrum resource has become more and more scarce. Cognitive radio (CR) technology [1][2][3] is a promising way to solve this problem. By sensing the unused spectrum in dynamically changing environments, the CR user, which is usually named the secondary user (SU), can opportunistically share the spectrum bands that may be assigned to the authorized user, which is usually named the primary user (PU). There are two paradigms for the secondary users to use the spectrum: underlay and overlay [4]. In the underlay mode, the SU may transmit data simultaneously with the PU, but the transmission power must be under certain threshold to avoid crucial interference with the PU system. In the overlay mode, the SUs can transmit data through the spectrum band that is not used at the moment by the PU system. In this paper, our system is using the overlay mode.

This work was supported by Sentech Chair in BWMC and DTI THRIP program.

S. Wang and B.T Maharaj are with the Department of Electrical, Electronic and Computer Engineering of University of Pretoria, Pretoria, South Africa. (email:ihsgnaw@gmail.com, sunil.maharaj@up.ac.za).

Attahiru S. Alfa is with the Department of Electrical, Electronic and Computer Engineering of University of Pretoria, Pretoria, South Africa and the Department of Electrical and Computer Engineering of University of Manitoba, Winnipeg, MB, Canada R3T 5V6. (email: attahiru.alfa@up.ac.za)
It is practical that the CR system is providing services to multiple users, and the system shares multiple spectrum bands with one or more PU systems. The way in which multiple PU channels are allocated to the SUs will definitely have significant influence on the quality of service $(\mathrm{Q} o \mathrm{~S})$ that the $\mathrm{CR}$ system can provide. There are many existing papers on how to evaluate the QoS performance of the CR system, among which the queueing analysis [5] is used often and has been proven to be efficient. By modeling the fading channel as finite-state Markov process [6], the data transmission process of CR system can be described by queueing model. In [7][8], from a cross-layer perspective, all the data transmitted in the CR system is modeled in a discrete form as packet, and a finite state Markov chain is used to describe the system with consideration of adaptive modulation and coding (AMC). Performance measures such as packet loss rate and average throughput are derived. In [9], automatic repeat request (ARQ) is considered and the delay statistics is also obtained. Then with similar queueing analysis, the effect of the PU channel allocation method on performance measures is investigated in [10]. Opportunistic spectrum scheduling is used to assign the unoccupied PU channels. However, no error recovery method is applied in the model. In [11], contention-based and contention-free medium access methods are applied to assign the channels.

The above channel allocation methods have some limitations. First, these methods are not configurable. The analytic model and the results of the performance evaluation are fixed once the methods are applied. Thus, not many adjustments of the channel allocation can be made to fit some of the performance measures to different requirements. For example, the opportunistic spectrum scheduling in [10], the PU channels are allocated to the SU that can transmit at the highest rate, thus one cannot guarantee any data transmission to the SU that cannot transmit at the same high rate as others, even when the SU with high rate has already transmitted all its data. Secondly, if the environmental parameters (for example the channel condition) change, the CR system will suffer from the the performance deterioration because of the unconfigurable allocation methods.

To provide a solution to the limitations above, the authors proposed the concept of a flexible channel allocation protocol, namely the distribution probability matrix (DPM), and preliminary analysis is carried out using Monte Carlo method in [12]. In this paper, the author proposed an analytical framework to carry out queueing analysis on the CR system with 
multiple channels and multiple users that applies the DPM as the channel allocation protocol. In the proposed framework, various environmental parameters and CR system settings are considered. In the spectrum sensing part, imperfect spectrum sensing [13][14] is assumed. The spectrum sensing parameters and the PU activity parameters are built into a spectrum sensing model to describe the relationship between SU sensing results and the occupancy states of the PU channels. In the channel allocation part, the allocation result is modeled as a Markov process by combining the spectrum sensing model and the DPM protocol. In the data transmission part, besides the AMC scheme adapted from [8] and [15], a truncated ARQ scheme [16] is applied. An analytic procedure is developed to describe the data transmission results under different situations using the AMC and truncated ARQ scheme. Finally, the authors build up a queueing model to describe the evolution of the $\mathrm{CR}$ system and various performance measures are derived. One advantage of the proposed framework is that all the processes considered are configurable to adapt various situations. Furthermore, even if one CR system is not exactly identical with one in this paper, estimated results can be provided by the proposed framework through fitting different settings with the parameters in the proposed model. After building up the queueing model, numerical results are presented to illustrate the impact of the DPM parameters, the impact of environmental parameters and the CR system settings on the performance measures. Further, the comparison of different channel allocation methods with DPM is carried out.

This paper is organized as follows. The system model and assumptions about the CR system is introduced in Section II. Then, how the queueing model is formulated is shown in Section III. Numerical results and the discussions about them are shown in Section IV. Section V concludes the paper.

\section{The System Model AND Assumptions}

\section{A. Network Model}

In this paper, an infrastructure-based cognitive radio network is considered, shown in Fig. 1. An SU system is working inside an area covered by a PU system. Both the PU and SU system are composed of one base station and multiple mobile stations. There is a reliable channel between the PU and SU base station to exchange all the necessary information including the channel condition, spectrum sensing results and synchronization information [17].

It is assumed that all the data transmitted in this system is in the form of packets, which are organized and buffered at the data link layer. The packets are transmitted within a physical layer frame. All the physical layer frames in the PU and SU systems are synchronized and of identical time duration $T_{\mathrm{f}}$, which is defined as one time slot. The spectrum band authorized to the PU system is divided into $M$ channels. The SU system shares the channels in the overlay mode: when the channel is not used by the PU system, the SU system can use it to transmit its packets. The data packets of SU system are stored in a finite buffer at each SU mobile station and the uplink data transmission from the SU mobile station to base station is considered in this paper.

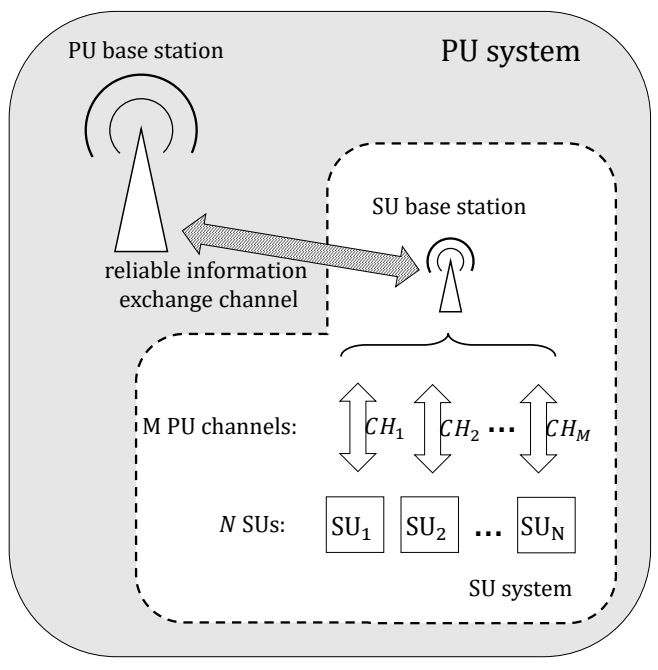

Fig. 1. System model.

\section{B. Primary User Activity}

The PU transmission activities on a channel are modeled as a time-homogenous first-order Markov process with two states. Let $O^{j}(t) \in\{0,1\}$ represent the PU occupancy state of the $j$-th channel at the $t$-th time slot. When the PU is transmitting, the state is called "busy" and $O^{j}(t)=0$, otherwise the state is called "free" and $O^{j}(t)=1$. The process can be described by a transition matrix $\boldsymbol{P}_{\mathrm{PU}}^{j}$ as:

$$
\boldsymbol{P}_{\mathrm{PU}}^{j}=\left(\begin{array}{cc}
p_{\mathrm{b} \rightarrow \mathrm{b}}^{j} & 1-p_{\mathrm{b} \rightarrow \mathrm{b}}^{j} \\
1-p_{\mathrm{f} \rightarrow \mathrm{f}}^{j} & p_{\mathrm{f} \rightarrow \mathrm{f}}^{j}
\end{array}\right),
$$

where $p_{\mathrm{b} \rightarrow \mathrm{b}}^{j}=\operatorname{Pr}\left\{O^{j}(t)=0 \mid O^{j}(t-1)=0\right\}$ denotes the probability that the $j$-th channel is occupied by PU (the "busy" state), given that the channel is occupied by PU (the "busy" state) at the previous time slot, and $p_{\mathrm{f} \rightarrow \mathrm{f}}^{j}=\operatorname{Pr}\left\{O^{j}(t)=\right.$ $\left.1 \mid O^{j}(t-1)=1\right\}$ denotes the probability that the $j$-th channel state changes from "free" to "free". These probabilities can be obtained by either measurement or estimation of the PU transmission behavior.

\section{Secondary System Transmission Model}

Each SU time slot consists of three successive parts: spectrum sensing, channel allocation and data transmission as shown in Fig. 2.

1) Spectrum sensing: At the beginning of each time slot, the SU system senses the existence of the PU transmission on every channel. Let $\hat{O}^{j}(t) \in\{0,1\}$ denote the sensing result. $\hat{O}^{j}(t)=1$ means the $\mathrm{SU}$ system estimates that the $j$-th channel is not occupied by PU system at the $t$-th time slot, and the SU system will allocate this channel to one of the SU mobile stations and transmit data between the base station and the mobile station. $\hat{O}^{j}(t)=0$ means the SU system estimates that the $j$-th channel is occupied by the PU system at the $t$-th time slot and the SU system will not use the channel during this time slot. In the proposed model, imperfect spectrum sensing is considered. There are 


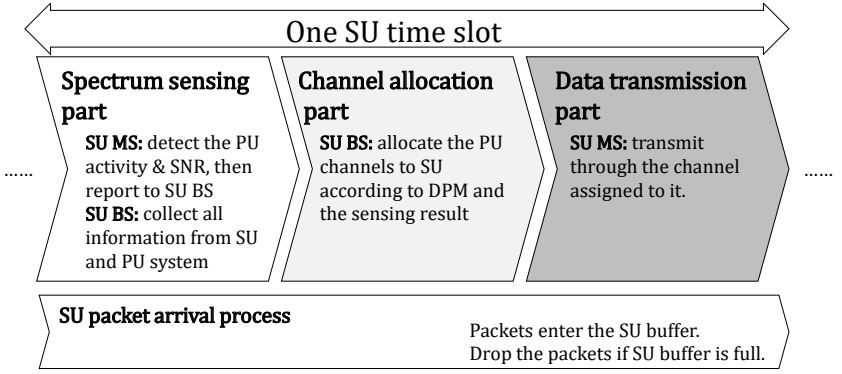

Fig. 2. Time slot structure of the secondary system.

two types of sensing errors: miss detection and false alarm. $P_{\mathrm{md}}=\operatorname{Pr}\left\{\hat{O}^{j}(t)=1 \mid O^{j}(t)=0\right\}$ represents the probability of miss detection, which means the SU system has the sensing result of a "free" state while the PU is actually transmitting. $P_{\mathrm{fa}}=\operatorname{Pr}\left\{\hat{O}^{j}(t)=0 \mid O^{j}(t)=1\right\}$ represents the probability of false alarm, which means the SU system has the sensing result of a "busy" state while the PU is not transmitting. If miss detection occurs, the packets the SU has transmitted on the channel in that time slot will interfere with the PU data transmission; these are defined as "collision" packets.

2) Channel Allocation: After the spectrum sensing part, the SU system allocates all the channels that are sensed "free" to the SU mobile stations. In this paper, a flexible and configurable protocol DPM the author developed in [12] is applied. The protocol works as follows: An $N$-by- $M$ distribution probability matrix is set up as:

$$
\boldsymbol{P}_{\mathrm{DPM}}=\left(\begin{array}{ccc}
p_{1}^{1} & \cdots & p_{1}^{M} \\
\vdots & \ddots & \vdots \\
p_{N}^{1} & \cdots & p_{N}^{M}
\end{array}\right)
$$

where $p_{i}^{j}$ represents the probability that the SU system allocates the $j$-th channel to the $i$-th $\mathrm{SU}$ if the channel is sensed "free". In the proposed framework, one SU can transmit through multiple channels during one time slot; meanwhile one PU channel can be accessed by only one SU. So in $\boldsymbol{P}_{\mathrm{DPM}}$ : $\sum_{j=1}^{M} P_{i}^{j}=1$ for any $i$.

3) Data transmission: In the data transmission part, AMC and truncated ARQ scheme are applied. The $j$-th PU channel is divided into $N_{\mathrm{CS}}^{j}$ states according to the signal-to-noise ratio (SNR) at the SU receiver. Let $c^{j}(t)=\left\{1,2, \cdots, N_{\mathrm{CS}}^{j}\right\}$ represent the channel condition state of the $j$-th channel at the $t$-th time slot. The evolution of the $j$-th channel condition states are modeled as a Markov chain using the same idea in [18]. The model is described by a $N_{\mathrm{CS}}^{j}$-by- $N_{\mathrm{CS}}^{j}$ transition matrix $\boldsymbol{P}_{\mathrm{CS}}^{j}$. According to the modulation method, the data transmission rate in every channel state can be obtained. Details are shown in Appendix A.

As the error recovery method, a truncated ARQ scheme shown in Fig. 3 is applied. It works as follows: If a packet is transmitted successfully, the transmitter will receive a ACK message from the receiver, otherwise, the receiver will send back an NAK message, and the transmitter will transmit the packet again. If a packet has been transmitted unsuccessfully for $L$ consecutive times, the transmitter will drop the packet,

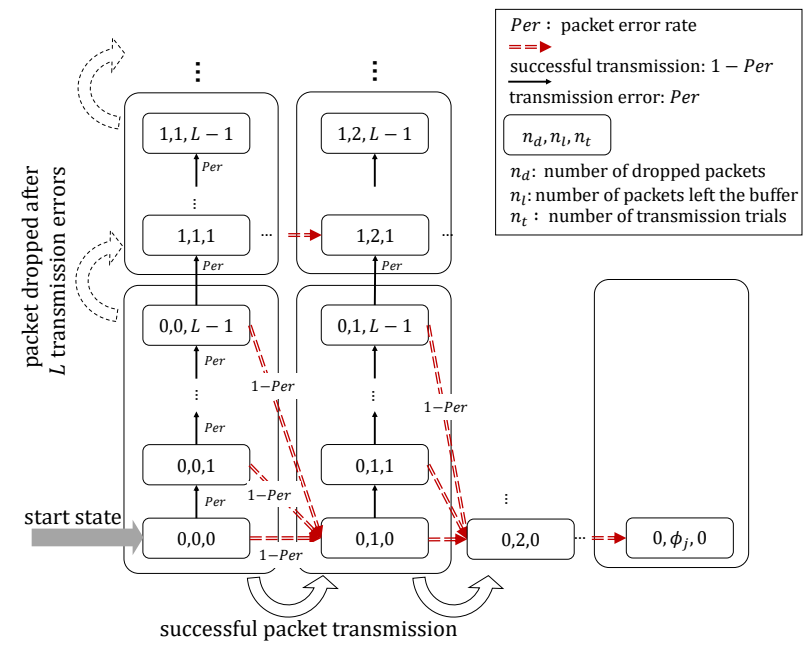

Fig. 3. The truncated ARQ model.

which is defined as a "drop" packet, then start to transmit the next packet. During the retransmission of the packets, specific technologies can be applied to improve the packet error rate, such as Maximal Ratio Combining (MRC) [19]. In the model of ARQ process in Appendix B, the packet error rate of the transmission and retransmissions can be configured according to the applied MRC method. To make the description of the proposed model concise, no further discussion on MRC is carried out, and in the following part of this paper, the packet error rates are assumed to be identical during the transmissions and retransmissions.

After data transmission, all packets arriving during the time slot will enter the buffer. If the total number of packets exceeds the buffer size, the overflow packets will be rejected. These packets are defined as "reject" packets.

\section{Formulation of The Queueing Model}

In order to confine the size of the proposed model, the following analytical model is built on one selected SU ( $i$-th). The procedure to analyze the other SUs in the system with the proposed model is identical.

\section{A. Arrival Process}

It is assumed that the number of arrival packets at each SU during one time slot follows a Batch Bernoulli process [20]. Let $\alpha_{i}(j)$ represents the probability that $j$ packets arrival during one time slot at the $i$-th $\mathrm{SU}$, and $v_{i}$ is the maximum number of packets that can arrive at the $i$-th SU during one time slot. Thus the process can be described by a probability vector $\boldsymbol{\alpha}_{i}$ as:

$$
\boldsymbol{\alpha}_{i}=\left\{\alpha_{i}(0), \alpha_{i}(1), \cdots, \alpha_{i}\left(v_{i}\right)\right\} .
$$

\section{B. Joint System States}

Besides the arrival packets, the SU transmission is affected by the channel assignment result and the transmission scheme. 


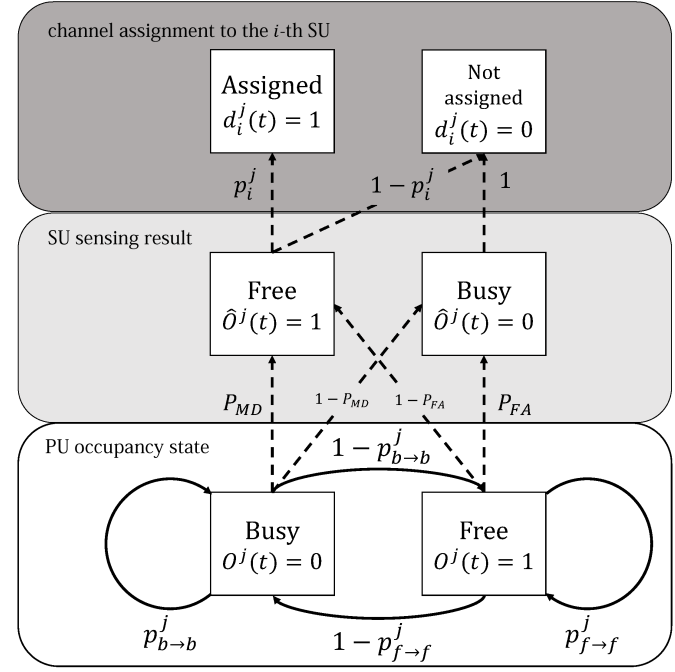

Fig. 4. Channel assignment state.

1) Channel assignment: In this model, a channel assignment state is used to describe whether the $\mathrm{SU}$ is allowed to transmit on the PU channels. Let $d_{i}^{j}(t)$ represent the assignment result, $d_{i}^{j}(t)=1$ represents that at the $t$-th time slot, the $j$-th channel is assigned to the $i$-th $\mathrm{SU}$, while $d_{i}^{j}(t)=0$ represents the opposite situation. Thus, a vector $\boldsymbol{d}_{i}^{j}(t)=\left\{\operatorname{Pr}\left(d_{i}^{j}(t)=0\right), \operatorname{Pr}\left(d_{i}^{j}(t)=1\right)\right\}$ can describe the possibility of the channel assignment state. Let the matrix $\boldsymbol{P}_{\hat{O}}$ describe the relationship between the actual PU occupancy state and SU sensing result. $\boldsymbol{P}_{\hat{O}}$ can be described by:

$$
\boldsymbol{P}_{\hat{O}}=\left(\begin{array}{cc}
1-P_{\mathrm{md}} & P_{\mathrm{md}} \\
P_{\mathrm{fa}} & 1-P_{\mathrm{fa}}
\end{array}\right) \text {. }
$$

Let $\boldsymbol{P} \boldsymbol{d}_{i}^{j}$ describe the relationship between SU sensing result and the channel assignment result, which can be obtained as follows:

$$
\boldsymbol{P d}_{i}^{j}=\left(\begin{array}{cc}
1 & 0 \\
1-p_{i}^{j} & p_{i}^{j}
\end{array}\right)
$$

where $p_{i}^{j}$ is the probability that the $j$-th channel is assigned to the $i$-th SU in (2). Using (4) and (5) the follows can be obtained:

$$
\boldsymbol{d}_{i}^{j}(t+1)=\boldsymbol{d}_{i}^{j}(t)\left(\boldsymbol{P}_{\hat{O}} \boldsymbol{P} \boldsymbol{d}_{i}^{j}\right)^{-1} \boldsymbol{P}_{\mathrm{PU}}^{j}\left(\boldsymbol{P}_{\hat{O}} \boldsymbol{P} \boldsymbol{d}_{i}^{j}\right),
$$

from which it can concluded that the assignment state of the $\mathrm{SU}$ is a Markov chain with the transition matrix $\boldsymbol{P}_{d}$, which can be obtained as follows:

$$
\boldsymbol{P}_{d}=\left(\boldsymbol{P}_{\hat{O}} \boldsymbol{P} \boldsymbol{d}_{i}^{j}\right)^{-1} \boldsymbol{P}_{\mathrm{PU}}^{j}\left(\boldsymbol{P}_{\hat{O}} \boldsymbol{P} \boldsymbol{d}_{i}^{j}\right) .
$$

The relationship among the PU occupancy state, SU sensing results and channel assignment results is shown in Fig. 4.

2) Joint channel state: The state of the $j$-th PU channel from the $i$-th SU's perspective can be described by the assignment state and the channel condition state. The state space $\boldsymbol{S}_{i}^{j}(t)$ can be defined as:

$\boldsymbol{S}_{i}^{j}(t) \triangleq\left\{d_{i}^{j}(t), c_{i}^{j}(t) \mid d_{i}^{j}(t) \in\{0,1\}, c_{i}^{j}(t) \in\left\{1,2, \cdots, N_{\mathrm{CS}}^{j}\right\}\right\}$.

Further, the joint state space $\boldsymbol{S}_{i}(t)$, which represents all possible channel conditions of $M$ PU channels from the $i$ th SU's perspective, can be written as:

$$
\begin{gathered}
\boldsymbol{S}_{i}(t) \triangleq\left\{\left(d_{i}^{1}(t), c_{i}^{1}(t)\right),\left(d_{i}^{2}(t), c_{i}^{2}(t)\right), \ldots,\left(d_{i}^{M}(t), c_{i}^{M}(t)\right) \mid\right. \\
\left.d_{i}^{j}(t) \in\{0,1\}, c_{i}^{j}(t) \in\left\{1,2, \cdots, N_{\mathrm{CS}}^{j}\right\}, j \in\{1,2, \cdots, M\}\right\} .
\end{gathered}
$$

It is assumed that all PU channel condition states to the $\mathrm{SU}$ are independent. According to the applied DPM and the activity of PU, the allocation state of PU channels is also independent. Hence, the number of all $M$ channel states is $N_{\text {all }}$ :

$$
N_{\text {all }}=2^{M} \cdot \prod_{j=1}^{M} N_{\mathrm{CS}}^{j} .
$$

An integer index $s_{i}(t)$ is set up to label each state in $\boldsymbol{S}_{i}(t)$ :

$$
s_{i}(t)=\sum_{j=1}^{M}\left\{\left[d_{i}^{j}(t) \cdot N_{\mathrm{CS}}^{j}+c_{i}^{j}(t)-1\right] \cdot \prod_{l=1}^{j} N_{\mathrm{CS}}^{l-1}\right\},
$$

where $N_{\mathrm{CS}}^{0}=0$ is set to make the expression concise. Then, the transition probability matrix of the channel state in which the states are sorted according to the indexes can be obtained:

$$
\boldsymbol{A}=\left(\boldsymbol{P}_{d}^{1} \otimes \boldsymbol{P}_{\mathrm{cs}}^{1}\right) \otimes \cdots \otimes\left(\boldsymbol{P}_{d}^{M} \otimes \boldsymbol{P}_{\mathrm{cs}}^{M}\right),
$$

where $\otimes$ denotes the Kronecker product.

\section{Service process}

Now, the detailed transmission process under any given system state $s_{i}(t) \in \boldsymbol{S}_{i}(t)$ can be obtained. The distribution of number of transmitted packets can be obtained, given the channel condition state considering the truncated ARQ and $\mathrm{AMC}$ scheme. Let $\Phi_{\text {all }}$ represent the maximum number of packets that can be transmitted during one time slot. A series of functions $\Theta$ can be used to describe the relationship between packets that can be transmitted and the channel state:

$$
\Theta(s(t))=\left\{\Theta_{0}(s(t)), \Theta_{1}(s(t)), \cdots, \Theta_{\Phi_{\text {all }}}(s(t))\right\},
$$

where $\Theta_{i}(s(t))$ represents the probability that $i$ packets can be transmitted successfully when the channel state is $s(t)$.

For ease of describing the service process, a matrix $\boldsymbol{\mu}_{i}$ is set up with the same size of $\boldsymbol{A}$, and it can be written as:

$$
\boldsymbol{\mu}_{i}=\left(\begin{array}{c}
\Theta_{i}(s(t)=1) \\
\vdots \\
\Theta_{i}\left(s(t)=N_{\text {all }}\right)
\end{array}\right) \otimes \mathbf{1}_{1 \times N_{\text {all }}},
$$

where $1_{1 \times N_{\text {all }}}$ is a 1-by- $N_{\text {all }}$ row vector whose elements are all 1. The details of the derivations of $\Theta$ and $\boldsymbol{\mu}_{i}$ are shown in Appendix B. 


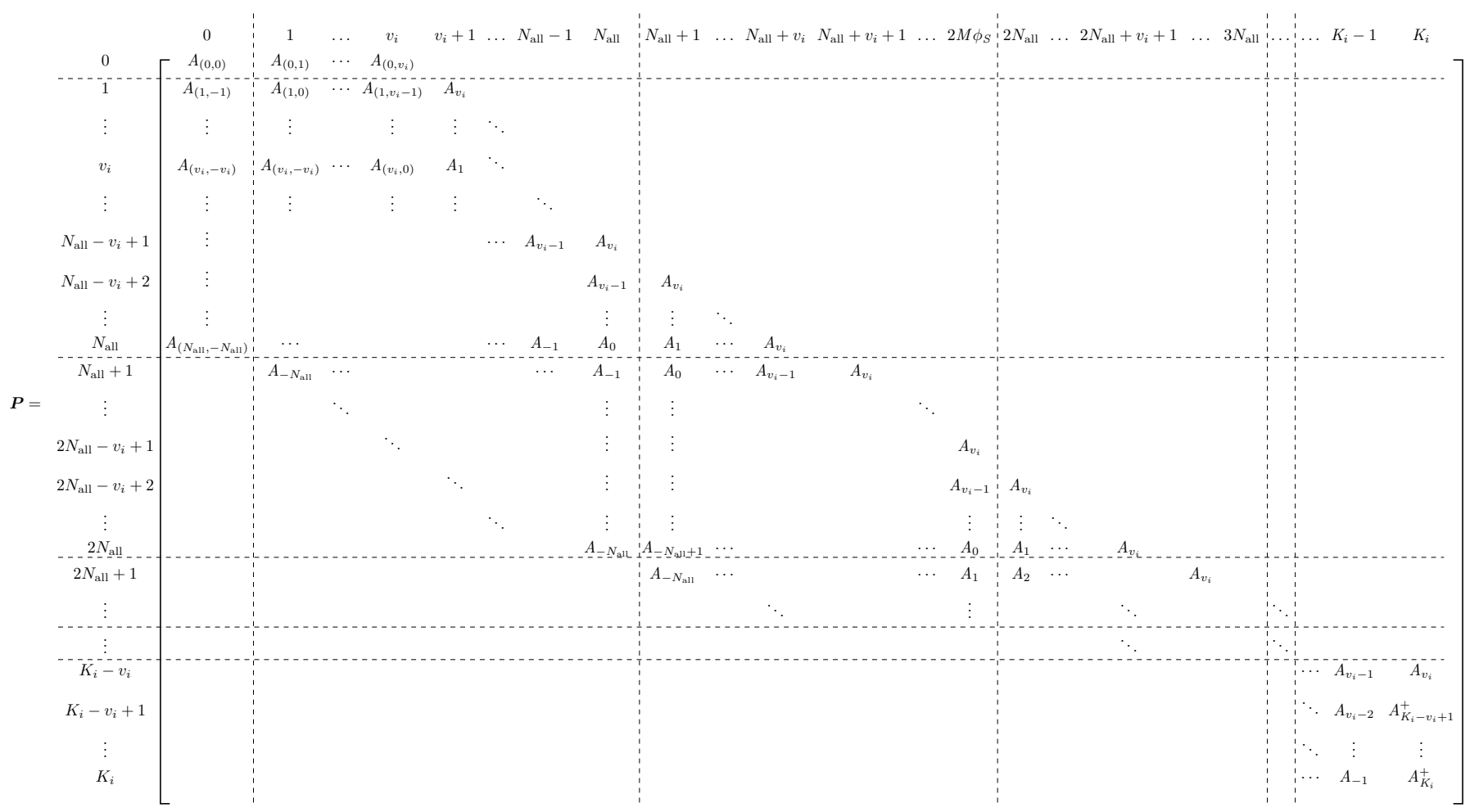

\section{Markov Chain Analysis}

The system state transitions happen from time slot to time slot so the system can be modeled as a discrete time Markov chain (DTMC). The buffer size of the $i$-th SU is $K_{i}$. The state space of the system can be set up as follows:

$\left\{\left(x_{i}(t), s_{i}(t)\right) \mid x_{i}(t) \in\left\{0,1, \cdots, K_{i}\right\} ; s_{i}(t) \in\left\{1,2, \cdots, N_{\text {all }}\right\}\right\}$

where $x_{i}(t)$ is the number of packets in the $i$-th SU's buffer at the $t$-th time slot, $s_{i}(t)$ denotes the channel condition state of all $M$ channels at the $t$-th time slot. With the arrival process described by $\boldsymbol{\alpha}_{i}$ and service process described by $\boldsymbol{\mu}$, the transition matrix $\boldsymbol{P}$ can be obtained and is shown in (??).

The inner matrix blocks of $\boldsymbol{P}$ are defined as follows:

$$
\boldsymbol{A}_{(0,0)}=\alpha_{i}(0) \cdot \boldsymbol{A}, \quad \boldsymbol{A}_{(0, k)}=\alpha_{i}(k) \cdot \boldsymbol{A}, \text { for } 1 \leq k \leq v_{i}
$$

$$
\begin{aligned}
& \boldsymbol{A}_{\Delta k}=\left(\sum_{m=\max (\Delta k, 0)}^{\min \left(\Delta k+N_{\text {all }}, v_{i}\right)} \alpha_{m} \boldsymbol{\mu}_{(m-\Delta k)}\right) \circ \boldsymbol{A}, \\
& \text { for }-N_{\text {all }} \leq \Delta k \leq v_{i} ; \\
& \boldsymbol{A}_{k}^{+}=\left(\sum_{n=l-k}^{v_{i}} \alpha_{n}\left(\sum_{m=0}^{\max (k-l+n, 0)} \boldsymbol{\mu}_{m}\right)\right) \circ \boldsymbol{A}, \\
& \text { for } K_{i}-v_{i}+1 \leq k \leq K_{i},
\end{aligned}
$$

where $\circ$ denotes the entrywise product. The other elements of $\boldsymbol{P}$ are all 0s.

\section{E. Steady State Distribution Vector}

The QR algorithm can be applied to the transition matrix to find the steady state probability of $\boldsymbol{P}$, which is represented by a vector $\pi$ :

$$
\boldsymbol{\pi}=\left\{\pi_{1}, \pi_{2}, \cdots, \pi_{\left(K_{i}+1\right) \times N_{\text {all }}}\right\},
$$

where $\pi_{i}$ is the $i$-th element of $\pi$. In order to evaluate the performance measures with ease, $\pi$ into $K_{i}+1$ vectors can be divided as:

$$
\boldsymbol{\pi}=\left\{\boldsymbol{\pi}_{0}, \boldsymbol{\pi}_{1}, \ldots, \boldsymbol{\pi}_{K_{i}}\right\}
$$

where:

$$
\boldsymbol{\pi}_{i}=\left\{\pi_{i \cdot N_{\text {all }}+1}, \pi_{i \cdot N_{\text {all }}+2}, \ldots, \pi_{(i+1) \cdot N_{\text {all }}}\right\}, 0 \leq i \leq K_{i},
$$

which indicates the probability of each joint channel state if there are $i$ packets in the queue.

\section{F. Performance Measures}

Using the steady state distribution vector, some performance measures of interest can be determined. In order to evaluate the QoS of SUs, and furthermore to evaluate the performance of the applied DPM to allocate the channels, the following performance measures are derived: average queue length, the packet rejecting rate, gross throughput, the packet collision rate, the packet dropping rate and average packet delay.

1) Queue Length: Let $q_{l}$ be the number of packets in queue. The probability of $l$ packets in queue can be obtained by summing up all elements in $\pi_{l}$ :

$$
\operatorname{Pr}\left(q_{l}=l\right)=\boldsymbol{\pi}_{l} \mathbf{1}=\sum_{m=l N_{\text {all }}+1}^{(l+1) N_{\text {all }}} \pi_{m}
$$


Therefore, the average queue length $Q_{l}$ can be obtained:

$$
Q_{l}=\sum_{m=0}^{K} m \operatorname{Pr}\left(q_{l}=m\right)
$$

From the average queue length, the buffer size the SU may need under certain circumstances can be investigated.

2) Packet Rejection Rate: At the beginning of an SU time slot, if the number of arrival packets plus the number of packets in queue after transmission exceeds the SU buffer size, the surplus packets are rejected. Let $r_{n}$ denote the probability that $n$ packets is rejected at the beginning of a time slot. $r_{n}$ can be obtained as :

$$
r_{n}=\sum_{m=n}^{v} \alpha_{i}(m)\left(\sum_{b=k-m+n}^{k} \boldsymbol{\pi}_{b}\left(\boldsymbol{\mu}_{b-k-n+m}\right)^{T}\right) .
$$

Hence the expected number of rejected packets during one time slot is :

$$
\operatorname{Rej}=\sum_{\mathrm{n}=1}^{\mathrm{v}} \mathrm{n} \cdot \mathrm{r}_{\mathrm{n}} .
$$

3) Gross Throughput: Gross throughput is indicated by the number of packets which leave the queue during one time slot, no matter these packets are successfully received, in collision with the PU or dropped. Let $\eta$ denote the average gross throughput, which can be obtained as:

$$
\begin{aligned}
\eta= & \sum_{m=1}^{N_{\text {all }}-1}\left(\sum_{a=1}^{m-1} a \boldsymbol{\pi}_{m} \boldsymbol{\mu}_{a}+m \boldsymbol{\pi}_{m} \sum_{b=m}^{N_{\text {all }}} \boldsymbol{\mu}_{b}\right) \\
& +\sum_{m=N_{\text {all }}}^{K} \sum_{a=1}^{N_{\text {all }}} a \boldsymbol{\pi}_{m} \boldsymbol{\mu}_{a} \\
= & \sum_{m=1}^{K} \sum_{a=1}^{N_{\text {all }}} \min (m, a) \boldsymbol{\pi}_{m} \boldsymbol{\mu}_{a} .
\end{aligned}
$$

4) Packet Collision Rate: When the miss detection happens in one time slot, all the packets transmitted by the SU on that channel will be in collision with the PU transmission. The expected number of collision packets can be derived as:

$$
\mathrm{Col}=\frac{\operatorname{Pr}\left(\mathrm{O}^{\mathrm{j}}(\mathrm{t})=0\right) \mathrm{P}_{\mathrm{md}}}{\operatorname{Pr}\left(\mathrm{O}^{\mathrm{j}}(\mathrm{t})=0\right) \mathrm{P}_{\mathrm{md}}+\left(1-\operatorname{Pr}\left(\mathrm{O}^{\mathrm{j}}(\mathrm{t})=0\right)\right)\left(1-\mathrm{P}_{\mathrm{fa}}\right)} \cdot \eta,
$$

where $\operatorname{Pr}\left(O^{j}(t)=0\right)$ is the probability of a channel being occupied by $\mathrm{PU}$ and can be derived as:

$$
\operatorname{Pr}\left(O^{j}(t)=0\right)=\frac{p_{\mathrm{b} \rightarrow \mathrm{b}}^{i}}{p_{\mathrm{b} \rightarrow \mathrm{b}}^{i}+p_{\mathrm{f} \rightarrow \mathrm{f}}^{i}} .
$$

5) Packet Dropping Rate: In order to derive the expected number of dropped packets, the percentage of dropped packets of all departure packets need to be calculated. From (37), the steady distribution of channel condition state of $j$-th channel $\pi_{\mathrm{cs} i}^{j}$ can be obtained. When channel is in state $k$, the expectation of number of dropped packets through channel $j$ is:

$$
E_{n_{d}}^{j}(i)=\sum_{a=1}^{N_{\text {all }}}\left(a \cdot \sum_{m=a \phi_{j} L}^{(a+1) \phi_{j} L-1} \psi_{m}\left(V_{\text {rate }}(k)\right)\right)
$$

where $\psi_{m}\left(V_{\text {rate }}(k)\right)$ represents the probability of $m$ dropped packets under ARQ state $k$. The expectation of number of packets departing through channel $j$ is:

$$
E_{n_{l}}^{j}(k)=\sum_{m=1}^{\phi_{j}} m \cdot \theta_{m}\left(V_{\text {rate }}(k)\right) .
$$

The details of $\psi_{m}\left(V_{\text {rate }}(k)\right)$ and $\theta_{m}\left(V_{\text {rate }}(k)\right)$ can be found in Appendix B, (50) and (51). Hence the packet dropping rate is:

$$
\rho_{\mathrm{drop}}=\sum_{j=1}^{M} p_{i}^{j} \frac{\sum_{m=1}^{N_{\mathrm{CS}}^{j}} E_{n_{d}}^{j}(m) \pi_{\mathrm{cs} i}^{j}(m)}{\sum_{m=1}^{N_{\mathrm{CS}}^{j}} E_{n_{l}}^{j}(m) \pi_{\mathrm{cs} i}^{j}(m)},
$$

where $p_{i}^{j}$ is the probability of the $j$-th channel allocated to the $i$-th SU. The expectation of number of dropped packets during one time slot is:

$$
\text { Drop }=\rho_{\text {drop }} \cdot \eta \text {. }
$$

6) Average Packet Delay: The average packet delay in this paper is defined as the expected time interval between the arrival of a packet and the start of its transmission. This interval consists of two parts: the transmission time of the packets which are in the buffer before packet arrival process and the transmission time of the packets which are arrived at the same time slot but transmitted before the packet of interest. Thus, the average packet delay can be obtained as:

$$
T_{w}=\frac{Q_{l}+\frac{\sum_{m=1}^{v_{i}} m \cdot \alpha_{i}(m)}{2}}{\eta} .
$$

\section{NumericAl Results AND Discussion}

In this paper, the configurable components of the analytical framework, such as the allocation protocol, the arrival process and the service process, are designed in a flexible way to adapt to as many scenarios as possible. However, due to the enormous size of the set of possible parameters, only some of the numerical results under typical parameters widely employed in the related literatures are presented to show how the performance measures of one selected SU change under different environmental parameters and CR system settings. The following are the default values of the parameters used in the performance evaluation:

- Number of PU channels: 2

- Number of SU: 2

- Packet length: $2 \mathrm{~ms}$

- Carrier frequency: $5 \mathrm{GHz}$

- Target error rate: $1 \%$

- PU activity settings: $p_{\mathrm{f} \rightarrow \mathrm{f}}^{1}=p_{\mathrm{f} \rightarrow \mathrm{f}}^{2}=p_{\mathrm{b} \rightarrow \mathrm{b}}^{1}=p_{\mathrm{b} \rightarrow \mathrm{b}}^{2}=$ 0.5

- Spectrum sensing settings: $P_{\mathrm{fa}}=0.00661, P_{\mathrm{md}}=$ 0.00661

- SU buffer size: $K_{i}=60$

- Packet arrival setting: $\boldsymbol{\alpha}_{i}=\{0.2,0.2,0.2,0.2,0.2\}$

- Average SNR of the two channels are both $10 \mathrm{~dB}$

- Channel condition settings are in Appendix A.

The following are investigated from the numerical results:

- How performance measures vary under different DPM parameters? 
- The impact of different environmental parameters and SU system settings on the relationship between performance measures and DPM parameters.

- Comparison of different channel allocation protocols.

\section{A. Performance Measures versus DPM Parameters}

In this part, the configurable feature of the DPM protocol and the performance measures that can be obtained under different DPM parameters are demonstrated. The channel allocation result is described by axis $p^{1}$ and axis $p^{2}$, which are the values of the probabilities that channel 1 and channel 2 are assigned to the $\mathrm{SU}$, respectively. In this model, the capacities of the PU channels are far better for the traffic of SUs, so the authors focus on the performance measures, which describe the data transmission failures and the demands of system settings. Fig. 5(a) shows the average number of packets in the SU buffer under different DPM parameters. It is clear and intuitive that if the SU has a higher chance of being allocated to PU channels (when $p^{1}$ and $p^{2}$ are bigger), the average number of packets in the buffer will decrease because the SU has a better chance of transmitting its packets. Fig. 5(b) shows the average number of collision packets in one time slot under different DPM parameters, which will increase when $p^{1}$ and $p^{2}$ are bigger because the more packets the SU transmits, the more collisions with the PU will occur. Fig. 5(c) shows that the average number of rejected packets under different DPM parameters. Its trend is same as average number of packets in the SU buffer because the fewer packets are in the buffer, the fewer packets will be rejected.

Further, when the environmental parameters vary, the proposed model can easily be adjusted to obtain the relationship between performance measures and DPM parameters. In Fig. 6, the average SNR at the SU receiver of the PU channel 2 is set to $30 \mathrm{~dB}$ and the relationship between performance measures and DPM parameters changes is shown. In Fig. 6(a) and Fig. 6(c), the average number of packets in the SU buffer and the average number of rejected packets under different DPM parameter are shown. Because the average SNR at the SU receiver of PU channel 2 is greater than the SNR of channel 1, the SU can transmit more packets through channel 2 . As a result, it is evident that the value of $p^{2}$ has greater impact on the performance measures than $p^{1}$ compared to Fig. 5(a) and Fig. 5(c). Fig. 6(b) shows the average number of collision packets in one time slot under different DPM parameters, and it can be discovered that the same trends of the number of collision packets under greater $p^{1}$ and $p^{2}$ as in Fig. 5(b). Further, as in Fig. 6(a) and Fig. 6(c), the value of $p^{2}$ has greater impact on the number of collision packets than $p^{1}$.

The relationship shown in the results obtained by the proposed framework may be used in the following ways:

- When designing a CR system, if there are some limits on certain performance measures (e.g. the data rate must be larger than a certain standard), the boundary of distribution probability of the SU that the applied channel allocation protocol must guarantee can be obtained.

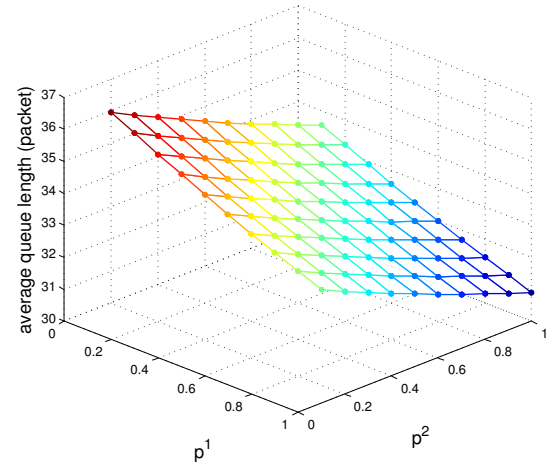

(a)

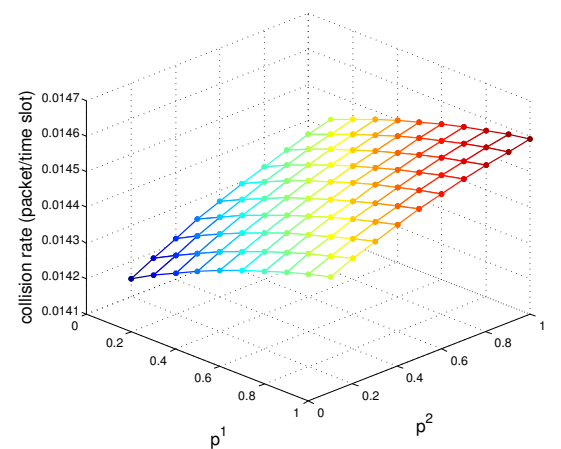

(b)

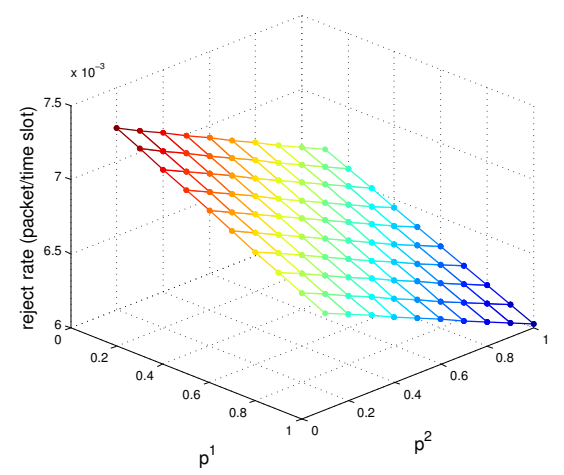

(c)

Fig. 5. Performance measures versus DPM parameters. The axis $p^{1}$ and axis $p^{2}$ are the distribution probabilities of the SU to channel 1 and channel 2 , respectively.

- Using the relationship between DPM parameters and performance measures (e.g. average number of packets in the buffer, average number of collision packets and average number of rejected packets), optimization of one performance measure or some of the performance measures combined can be carried out easily.

- If the CR system is sharing different channels or even channels from a different kind of system with different parameters, the proposed allocation protocol can be adjusted accordingly to fulfill the performance requirements or optimize the performance.

\section{B. Impact of System Settings on Performance Measures}

In this part, the impact of the buffer size $K_{i}$ and the truncated ARQ setting $L$ on the performance measures is 


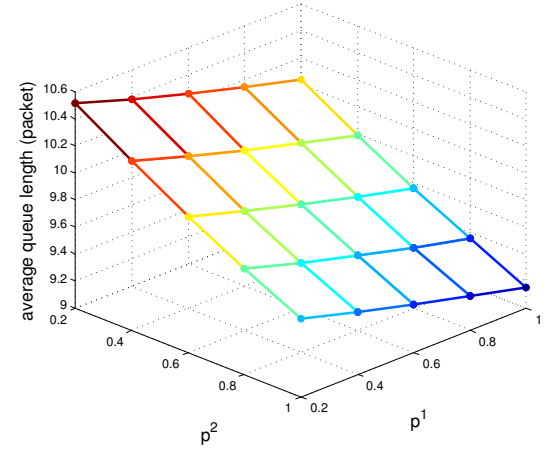

(a)

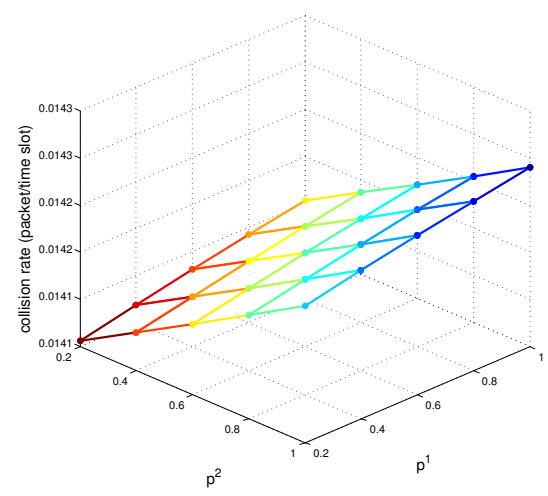

(b)

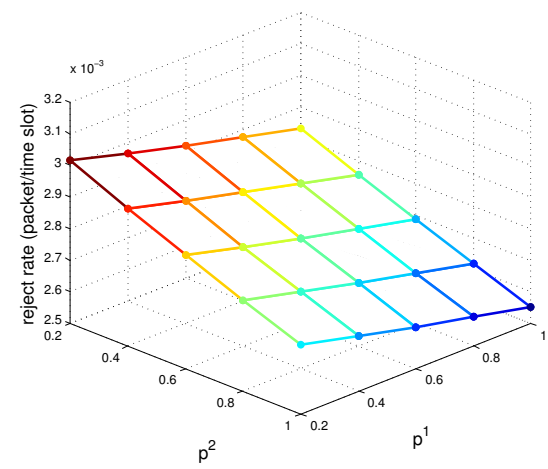

(c)

Fig. 6. Performance measures versus DPM parameters with different SNRs The SNR of PU channel 1 is $10 \mathrm{~dB}$, and the SNR of PU channel 2 is $30 \mathrm{~dB}$.

investigated to demonstrate how this model can be used to help design CR system settings to meet the performance requirements. In Fig. 7, the buffer size of the $\mathrm{SU}$ is set from 40 to 60 and changes in the performance measures are investigated under the DPM parameters: $p^{1}=p^{2}=0.5$. In Fig. 7(a), the average packet waiting time increases when the SU buffer size becomes larger because larger buffer size will cause more packets waiting in the buffer. Fig. 7(b) shows that the number of rejected packets will decrease because the probability that the buffer becomes full is less when the buffer size is larger.

In Fig. 8, the truncated ARQ parameter $L$ is set from 3 to 8 and the impact on the number of dropped packets and average waiting time under different SU arrival patterns and channel conditions is investigated. The packet dropping rate is affected

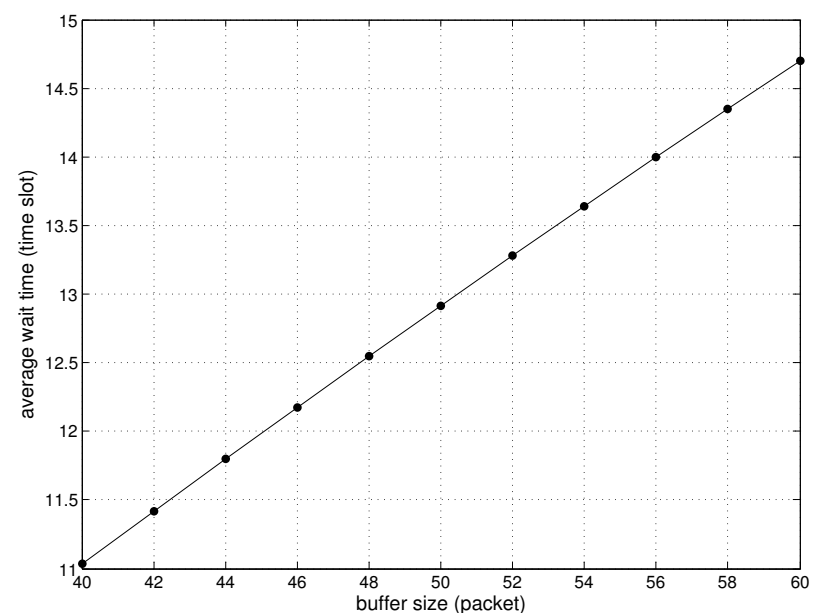

(a)

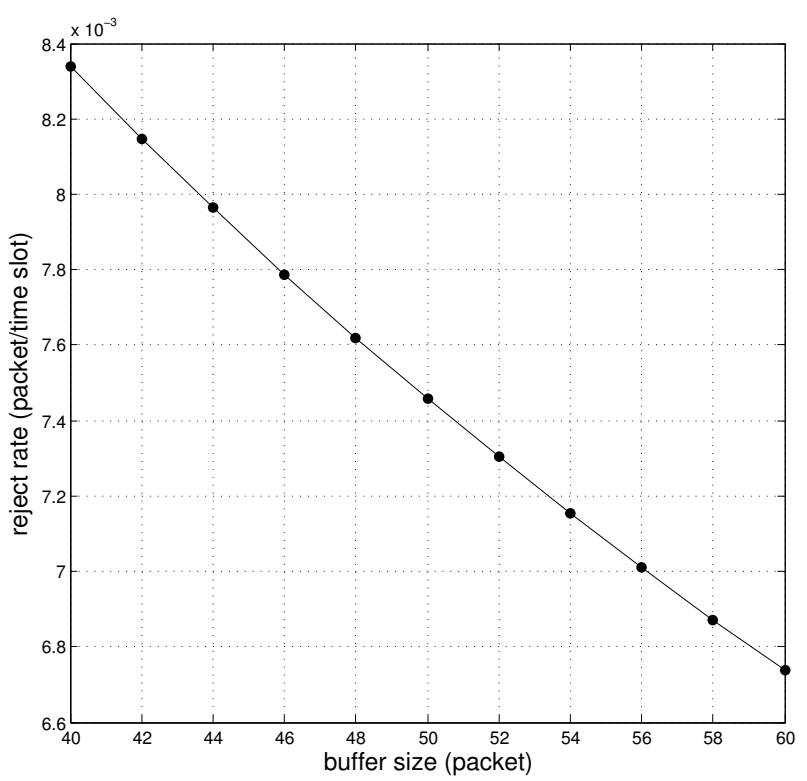

(b)

Fig. 7. Performance measures versus SU buffer size.

mainly by the ARQ parameter $L$ and almost unaffected by the arrival rate and the channel conditions. The packet average waiting time is increased by $L$ when $L$ is less than 5 ; after that there is no distinct change in the waiting time with the increase in $L$.

The relationship between the $\mathrm{CR}$ system settings and selected performance measures revealed by this model can help analyze which CR system settings are the key factor influencing particular performance measures. Further, the CR system can be designed properly to meet the selected performance requirements.

\section{Comparison between Different Channel Allocation Meth- ods}

In this part, the DPM with the maximum rate (MR) scheme and random allocation scheme are compared. The MR scheme in this paper works as follows: When a PU channel is not occupied and is available to the SU system, the SU system 


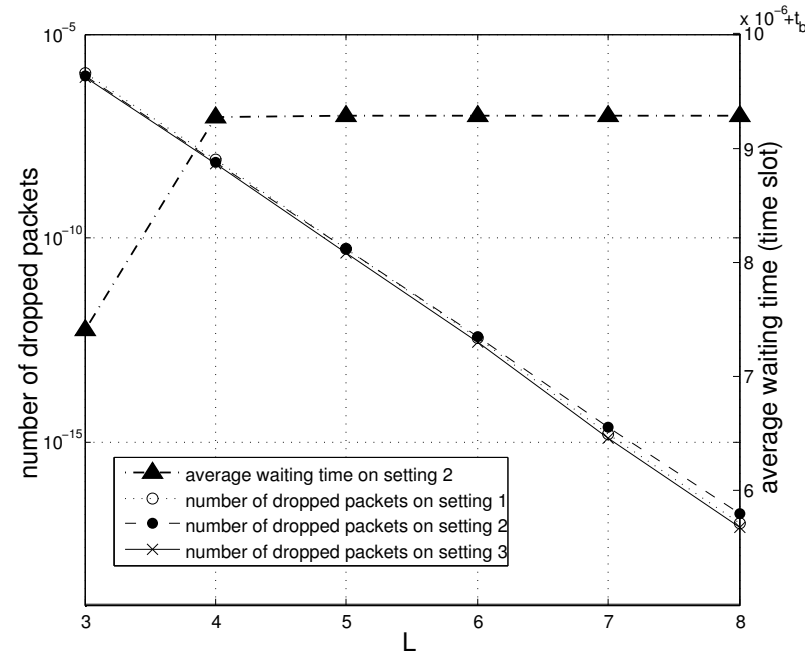

Fig. 8. The impact of ARQ parameter $L$ on performance measures under different system settings. $t_{b}=11.03447$. Setting 1: SU packet arrival vector: $\boldsymbol{\alpha}=\{0.2,0.2,0.2,0.2,0.2\}$, SNR of PU channel 1 and 2: $10 \mathrm{~dB}$ Setting 2: $\boldsymbol{\alpha}=\{0.2,0.4,0.4\}$, SNR of PU channel 1 and 2: $10 \mathrm{~dB}$. Setting 3: $\boldsymbol{\alpha}=\{0.2,0.4,0.4\}$, SNR of PU channel 1 and 2: $30 \mathrm{~dB}$.

will assign it to the SU that can transmit at the highest rate. In the random allocation scheme, all channels are allocated randomly to each SU with the same probabilities. In a CR system of $N$ SUs and $M$ channels, the random allocation scheme can be represented by setting the DPM parameter matrix as $p_{i}^{j}=1 / M$ for $i \in\{1, \ldots, N\} 1, j \in\{1, \ldots, M\}$. The main difference in the methods is the way in which PU channels are allocated to the SUs after the SU system has collected all necessary information from the PU and SU systems. In order to compare the complexities of the allocation procedure under these methods, the time costs of channel allocation computation of the three channel allocation methods by MATLAB simulation are presented in Table I. Then, the performance under different channel allocation methods is compared. Fig. 9 shows the average number of rejected packets in one time slot when the DPM parameters are $p^{1}=p^{2}$ and $p^{1}, p^{2} \in\{0.1,0.2, \ldots, 1\}$ and the comparison with the average number of rejected packets under the MR scheme and random allocation scheme. When the parameters are set properly $\left(p^{1}, p^{2}>0.5\right)$, the DPM can outperform the random allocation. The optimal average number of rejected packets under the DPM is close to the number under the MR scheme. However, if the MR scheme is applied, it will cost the SU system more resources to compare the channel condition and the data rate of the SU mobile stations than DPM and random allocation as shown in Table I. Furthermore the accuracy of the results of the comparison will affect the practical performance. So when designing the channel allocation schemes, there is a trade-off between the complexity and the performance to be considered, and the proposed framework can provide references for that.

\section{Conlusions}

The authors have developed a configurable channel allocation protocol which is able to describe the allocation
TABLE I

Time Costs of Channel Allocation Computation

\begin{tabular}{|l||c|c|c|}
\hline \multicolumn{1}{|c||}{ Parameters } & \multicolumn{3}{c|}{ Time cost ratio to DPM } \\
\cline { 2 - 4 } & DPM & MR & random allocation \\
\hline \hline $\begin{array}{l}\text { number of SU:2 } \\
\text { number of channels: } 2\end{array}$ & 1 & 10.8 & 0.24 \\
\hline $\begin{array}{l}\text { number of SU:2 } \\
\text { number of channels: } 10\end{array}$ & 1 & 11 & 0.22 \\
\hline $\begin{array}{l}\text { number of SU:4 } \\
\text { number of channels: } 10\end{array}$ & 1 & 9.1 & 0.26 \\
\hline
\end{tabular}

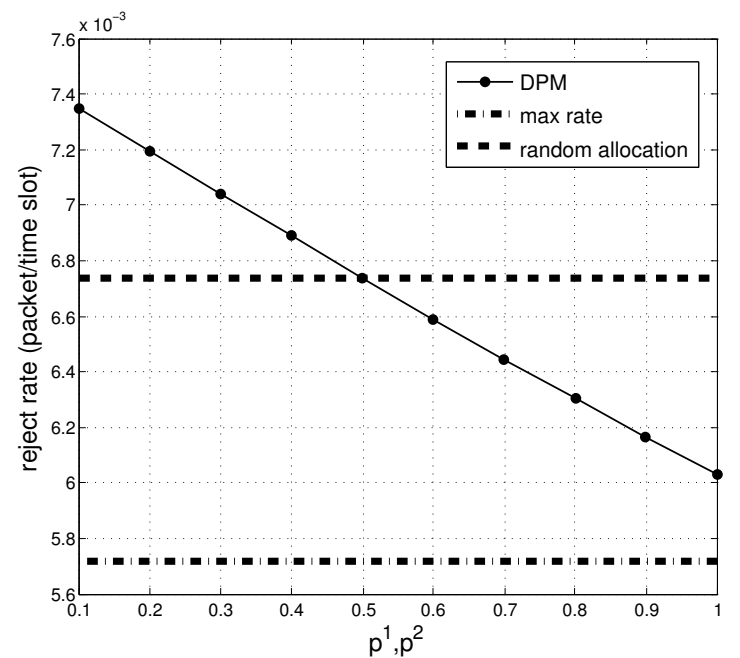

Fig. 9. Comparison of average queue length and reject rate of different channel allocation methods.

result more comprehensively than the other fixed channel allocation protocols, as in [10] and [11]. A queueing analytic framework to study how the channel allocation results under different environmental parameters and different CR system settings affect the system performance measures is presented. Important performance measures have been derived by fitting the link adaptation technologies, such as AMC and truncated ARQ and the proposed channel allocation protocol (DPM), into the queueing model. The influence of CR system settings on performance measures in different channel allocation situations described by the DPM is investigated. Through the presented results the DPM parameters that can achieve optimal performance can be obtained. The comparison of channel allocation protocols shows that firstly, the protocol outperforms the random allocation protocol, secondly the proposed protocol is able to yield performance close to the MR protocol, which costs the system more resources. Our framework can be applied to design or evaluate new CR channel allocation protocols and to provide references to determine the SU system settings to achieve the performance requirements.

\section{APPENDIX A}

\section{CHANNEL STATE MODEL}

When the PU channel is available to SUs, an adaptive modulation and coding (AMC) scheme is used to make better use of the channel resources [21]. In this paper, the transmission mode on the physical layer is convolutionally coded $M_{n}$-ary rectangular or square QAM modes, which are adopted from 
the HIPERLAN/2 or IEEE 802.11a standards [15]. The details of the AMC scheme are shown in Table II.

The state of the $j$-th channel condition is divided into $N_{\mathrm{CS}}^{j}$ parts. Different modulation schemes are used in each part to make the packet error rates of each part all equal to a required packet error rate $P_{\text {target }}$. Thus, the state boundary in terms of SNR can be written in [7] as:

$$
\Gamma_{n}=\frac{1}{g_{n}} \ln \left(\frac{a_{n}}{P_{\text {target }}}\right),
$$

where $\Gamma_{n} \in\left\{\Gamma_{0}, \Gamma_{1}, \ldots, \Gamma_{N_{\mathrm{CS}}^{j}}\right\}$ denotes the boundary SNR of each state. Then by setting $\Gamma_{0}=0$ and $\Gamma_{N_{\mathrm{CS}}^{j}+1}=\infty$, the $N_{\text {CS }}^{j}+1$ SNR boundary of the $N_{\text {CS }}^{j}$ states can be obtained. When the SNR at an SU's receiver satisfies $\gamma \in\left(\Gamma_{0}, \Gamma_{1}\right)$, the SU does not transmit any packets because of the low SNR and when $\gamma \in\left(\Gamma_{n}, \Gamma_{n+1}\right), n \in\left\{1,2, \ldots, N_{\mathrm{CS}}^{j}-1\right\}$, the SU is transmitting using mode $n$.

Then one can determine how the channel condition evolves from time slot to time slot. It is assumed that the channel is Rayleigh: the SNR is exponentially distributed with the probability density function:

$$
p\left(\gamma_{i}^{j}\right)=\frac{1}{\bar{\gamma}_{i}^{j}} \exp \left(-\frac{\gamma_{i}^{j}}{\bar{\gamma}_{i}^{j}}\right), \quad \gamma_{i}^{j} \geq 0
$$

where $\gamma_{i}^{j}$ is the received SNR of the $i$-th SU on the $j$ th channel, and $\bar{\gamma}_{i}^{j}$ is the average SNR. The steady state probability of $i$-th SU on the $j$-th channel in state $k$ can be obtained by:

$$
\pi_{\mathrm{CSi}}^{j}(k)=\int_{\Gamma_{k}}^{\Gamma_{k+1}} p\left(\gamma_{i}^{j}\right) \mathrm{d} x .
$$

Let $\boldsymbol{P}_{\mathrm{CSi}}^{j}=\left(\begin{array}{ccc}p_{0,0} & \cdots & p_{0, N_{\mathrm{CS}}^{j}-1} \\ \vdots & \ddots & \vdots \\ p_{N_{\mathrm{CS}}^{j}-1,0} & \cdots & p_{N_{\mathrm{CS}}^{j}-1, N_{\mathrm{CS}}^{j}-1}\end{array}\right)$ denote the state transition probability matrix of the $i$-th SU on the $j$ th channel, where $p_{a, b}$ denotes the transition probability from state $a$ to state $b$. According to [22], the transition probabilities can be obtained by:

$$
\begin{gathered}
p_{k, k+1} \approx \frac{f_{m} T_{p}}{\pi_{\mathrm{CSi}}^{j}(k)} \sqrt{\frac{2 \pi \Gamma_{k+1}}{\bar{\gamma}_{i}^{j}}} \exp \left(-\frac{\Gamma_{k+1}}{\bar{\gamma}_{i}^{j}}\right), \\
p_{k, k-1} \approx \frac{f_{m} T_{p}}{\pi_{\mathrm{CSi}}^{j}(k)} \sqrt{\frac{2 \pi \Gamma_{k}}{\bar{\gamma}_{i}^{j}}} \exp \left(-\frac{\Gamma_{k}}{\bar{\gamma}_{i}^{j}}\right),
\end{gathered}
$$

where $f_{m}$ is the maximum Doppler frequency. It is assumed that the transition occurs only between adjacent states: $p_{j, k}=$ 0 , for any $|j-k|>1$, and $p_{k, k}=1-p_{k, k+1}-p_{k, k-1}$, then with (35)-(39), the whole transition probability matrix $\boldsymbol{P}_{\mathrm{CS}}^{j}$ is obtained. Let $V_{\text {rate }}(k)$ represent the number of packets that can be transmitted in one time slot when the channel condition state is $k$. According to the rate in Table II, $\left\{V_{\text {rate }}(k), k \in\right.$ $\{0,1, \cdots, 6\}\}=\{0,2,4,6,9,12,16\}$.
TABLE II

TRANSMISSION MODES WITH CONVOLUTIONALLY CODED MODULATION

\begin{tabular}{c||c|c|c|c|c|c}
\hline & Mode 1 & Mode 2 & Mode 3 & Mode 4 & Mode 5 & Mode 6 \\
\hline \hline Modulation & BPSK & QPSK & 8-QAM & 16-QAM & 32-QAM & 64-QAM \\
\hline Coding rate $R_{c}$ & $1 / 2$ & $1 / 2$ & $3 / 4$ & $9 / 16$ & $3 / 4$ & $3 / 4$ \\
\hline Rate(bits/symbol) & 0.5 & 1 & 1.5 & 2.25 & 3 & 4 \\
\hline$a_{n}$ & 274.7229 & 90.2514 & 67.6181 & 50.1222 & 53.3987 & 35.3508 \\
\hline$g_{n}$ & 7.9932 & 3.4998 & 1.6883 & 0.6644 & 0.3756 & 0.09 \\
\hline$\Gamma_{n}(\mathrm{~dB})$ & -1.5331 & 1.0942 & 3.9722 & 7.7021 & 10.2488 & 15.9784 \\
\hline \multicolumn{7}{|r|}{}
\end{tabular}

\section{APPENDIX B}

\section{TRANSMISSION PROCESS}

It can be obtained from AMC settings that there are total $\phi_{j}$ transmission attempts in one SU time slot from the $j$-th channel. Let $n_{l} \in\left\{0,1, \ldots, \phi_{j}\right\}$ denote number of packets leaving the queue from the channel, $n_{t} \in\{0,1, \ldots, L-1\}$ denote number of transmission trials and $n_{d} \in\left\{0,1, \ldots,\left\lfloor\frac{\phi_{j}}{L}\right\rfloor\right\}$ denote number of packets dropped because of a full SU buffer. A state space $\left\{n_{d}, n_{l}, n_{t}\right\}$ can be built then, which is called the ARQ state, and derive the transition matrix $\boldsymbol{P}_{\mathrm{ARQ}}$. The transition matrix $\boldsymbol{P}_{\mathrm{ARQ}}$ consists of two levels of matrix blocks. The block of first level describes the transition between number of transmission trials. $\boldsymbol{P}_{0}$ describes the state of the transition when the transmission has failed:

$$
\boldsymbol{P}_{0}=\begin{array}{ccccc}
0 & 1 & 2 & \ldots & L-1 \\
& \vdots \\
& L-2 \\
L-1 &
\end{array}\left(\begin{array}{ccccc}
0 & \text { Per } & & & \\
& 0 & \text { Per } & & \\
& & \ddots & \ddots & \\
& & & 0 & \text { Per } \\
& & & 0 & 0
\end{array}\right)
$$

$\boldsymbol{P}_{1}$ describes the state of the transition when the transmission has been successful:

$$
\begin{aligned}
& \begin{array}{llll}
0 & 1 & \ldots & L-1
\end{array} \\
& \boldsymbol{P}_{1}=\begin{array}{l}
0 \\
\vdots \\
L-1
\end{array}\left(\begin{array}{cccc}
1-\text { Per } & 0 & \ldots & 0 \\
1-\text { Per } & 0 & \ldots & 0 \\
\vdots & \vdots & \ddots & 0 \\
1-\text { Per } & 0 & \ldots & 0
\end{array}\right)
\end{aligned}
$$

$\boldsymbol{P}_{\text {drop }}$ describes the state of the transition when a packet has been dropped after consecutive $L$ failed transmission attempts:

$$
\left.P_{\text {drop }}=\begin{array}{cccc}
0 & 1 & \ldots & L-1 \\
0 \\
\vdots-1 \\
0 & 0 & \ldots & 0 \\
0 & 0 & \ldots & 0 \\
\vdots & \vdots & \ddots & 0 \\
\text { Per } & 0 & \ldots & 0
\end{array}\right) .
$$

Here, if the MRC method is applied, the Per can be modified accordingly to adapt the improvement on packet error rate during packet retransmissions.

The block of the second level describes the transition between number of departure packets. $\boldsymbol{Q}_{i}$ describes the transition 
between number of departure packets when $i$ packets have been dropped:

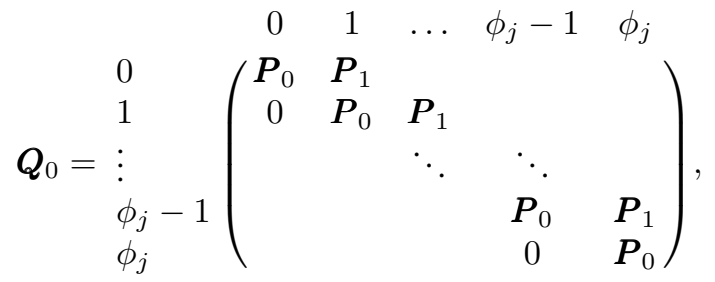

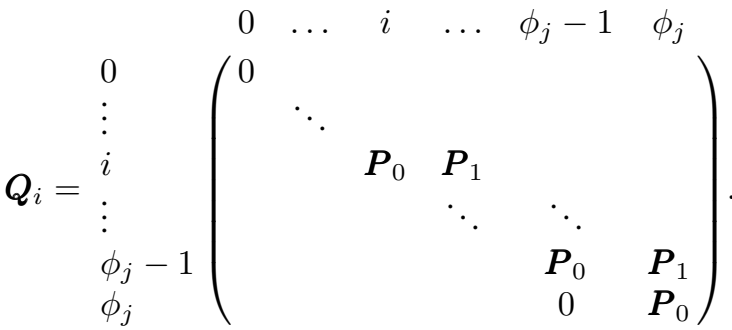

$D_{i}$ describes the transition when a new packet is dropped when $i$ packets have been dropped:

$$
\boldsymbol{D}_{0}=\begin{array}{ccccc}
0 & 1 & \cdots & \phi_{j}-1 & \phi_{j} \\
0 & \vdots \\
\phi_{j}-1 & \phi_{j}
\end{array}\left(\begin{array}{ccccc}
0 & \boldsymbol{P}_{\text {drop }} & & & \\
& 0 & \boldsymbol{P}_{\text {drop }} & & \\
& & \ddots & \ddots & \\
& & & 0 & \boldsymbol{P}_{\text {drop }} \\
& & & 0 & 0
\end{array}\right)
$$

$$
\begin{aligned}
& \begin{array}{lllllll}
0 & \ldots & i & i+1 & \ldots & \phi_{j}-1 & \phi_{j}
\end{array} \\
& \begin{array}{l}
0 \\
\boldsymbol{D}_{i}= \\
\vdots \\
i \\
i+1 \\
\vdots \\
\phi_{j}-1 \\
\phi_{j}
\end{array}\left(\begin{array}{ccccccc}
0 & & & & & & \\
& \ddots & & & & & \\
& & 0 & \boldsymbol{P}_{\text {drop }} & & & \\
& & 0 & \boldsymbol{P}_{\text {drop }} & & \\
& & & & \ddots & \ddots & \\
& & & & 0 & \boldsymbol{P}_{\text {drop }} \\
& & & & 0 & 0
\end{array}\right)
\end{aligned}
$$

Finally, the transition matrix can be obtained:

$$
\boldsymbol{P}_{\mathrm{ARQ}}=\left(\begin{array}{ccccc}
\boldsymbol{Q}_{0} & \boldsymbol{D}_{0} & & & \\
& \boldsymbol{Q}_{1} & \boldsymbol{D}_{1} & & \\
& & \ddots & \ddots & \\
& & & \boldsymbol{Q}_{\left\lfloor\frac{\phi_{j}}{L}\right\rfloor-1} & \boldsymbol{D}_{\left\lfloor\frac{\phi_{j}}{L}\right\rfloor-1} \\
& & & & \boldsymbol{Q}_{\left\lfloor\frac{\phi_{j}}{L}\right\rfloor}
\end{array}\right)
$$

Let $\Phi=\left\lfloor\frac{\phi_{S}}{L}\right\rfloor, \boldsymbol{P}_{A R Q}$ is a $\left((\Phi+1) \cdot\left(\phi_{j}+1\right) \cdot L\right) \times$ $\left((\Phi+1) \cdot\left(\phi_{j}+1\right) \cdot L\right)$ matrix. The transition diagram is shown in Fig. 3. A series of 1-by- $\left((\Phi+1) \cdot\left(\phi_{j}+1\right) \cdot L\right)$ vector $\boldsymbol{b}_{i}$ is used to represent the probability distribution of ARQ state $\left\{n_{d}, n_{l}, n_{t}\right\}$ after $i$ transmissions. Let $b_{i}\left(I\left(n_{d}, n_{l}, n_{t}\right)\right)$ denote the $I\left(n_{d}, n_{l}, n_{t}\right)$-th element of $\boldsymbol{b}_{i}$, which indicates the probability that the ARQ state is $\left\{n_{d}, n_{l}, n_{t}\right\}$ :

$$
I\left(n_{d}, n_{l}, n_{t}\right)=n_{t}+n_{l} \cdot L+n_{d} \cdot L\left(\phi_{j}+1\right)+1 .
$$

At the beginning of transmission, the ARQ state starts at $\{0,0,0\}$ which is represented by $\boldsymbol{b}_{0}=\{1,0,0, \ldots\}$. Then $\boldsymbol{b}_{i}$ which represents the distribution of the ARQ state after $i$ transmissions can be obtained as:

$$
\boldsymbol{b}_{i}=\boldsymbol{b}_{0} \cdot\left(\boldsymbol{P}_{\mathrm{ARQ}}\right)^{i} .
$$

From $\boldsymbol{b}_{i}$ and (48), The distribution of the number of packets leaving the buffer and the number of packets dropped can be obtained:

$$
\begin{gathered}
\psi_{m}(i)=\sum_{j=(m-1) L\left(\phi_{j}+1\right)+1}^{m L\left(\phi_{j}+1\right)} b_{i}(j), 0 \leq m \leq \Phi \\
\theta_{m}(i)=\sum_{a=0}^{\Phi}\left(\sum_{j=a L\left(\phi_{j}+1\right)+m L+1}^{a L\left(\phi_{j}+1\right)+(m+1) L+1} b_{i}(j)\right), 0 \leq m \leq \phi_{j},
\end{gathered}
$$

where $\psi_{m}(i)$ is the probability that $m$ packets are dropped after $i$ transmissions and $\theta_{m}(i)$ is the probability of $m$ packets leaving the queue after $i$ transmissions.

With (51), the distribution of the total number of departure packets transmitted through all $M$ channels can be obtained, given the joint states: $s(t)$. A series of functions $C^{j}$ are set up, and using $V_{\text {rate }}$, (11), the number of the transmission on the $j$-th channel when the channel state is $s(t), C^{j}(s(t))$ can be derived. Then, the distribution of departure packets when the channel state is $s(t)$ can be obtained, which is presented by $\boldsymbol{\Theta}(s(t))$ :

$\boldsymbol{\Theta}(s(t))=\boldsymbol{\theta}^{1}\left(C^{1}(s(t))\right) \circ \boldsymbol{\theta}^{2}\left(C^{2}(s(t))\right) \circ \cdots \circ \boldsymbol{\theta}^{M}\left(C^{M}(s(t))\right)$,

where $\circ$ denotes the convolution of vectors. $\boldsymbol{\Theta}(s(t))$ has $N_{\text {all }}+1$ elements. Let $\Theta_{i}(s(t))$ denote the $i$-th element of $\Theta(s(t))$, which is the probability of $i$ departure packets given the joint state $s(t)$. The $\boldsymbol{\Theta}(s(t))$ can be obtained given all possible $N_{\text {all }}$ different $s(t)$. Thus, a matrix $\boldsymbol{\mu}_{i}$, which represents the probability of $i$ departure packets given all possible channel states $s(t)$ in sequence, can be set up:

$$
\boldsymbol{\mu}_{i}=\left(\begin{array}{c}
\Theta_{i}(s(t)=1) \\
\vdots \\
\Theta_{i}\left(s(t)=N_{\text {all }}\right)
\end{array}\right) \otimes \mathbf{1}_{1 \times N_{\text {all }}} .
$$

where $1_{1 \times N_{\text {all }}}$ is a 1 -by- $N_{\text {all }}$ row vector whose elements are all 1.

\section{REFERENCES}

[1] J. Mitola III and G. Q. Maguire Jr, "Cognitive radio: making software radios more personal," Personal Communications, IEEE, vol. 6, no. 4, pp. 13-18, 1999.

[2] S. Haykin, "Cognitive radio: brain-empowered wireless communications," Selected Areas in Communications, IEEE Journal on, vol. 23, no. 2, pp. 201-220, 2005 .

[3] A. Goldsmith, S. A. Jafar, I. Marić, and S. Srinivasa, "Breaking spectrum gridlock with cognitive radios: an information theoretic perspective," Proceedings of the IEEE, vol. 97, no. 5, pp. 894-914, 2009.

[4] I. F. Akyildiz, W.-Y. Lee, M. C. Vuran, and S. Mohanty, "Next generation/dynamic spectrum access/cognitive radio wireless networks: a survey," Computer Networks, vol. 50, no. 13, pp. 2127-2159, 2006.

[5] A. S. Alfa, Queueing theory for telecommunications: discrete time modelling of a single node system. Springer Science \& Business Media, 2010. 
[6] P. Sadeghi, R. A. Kennedy, P. B. Rapajic, and R. Shams, "Finitestate Markov modeling of fading channels - a survey of principles and applications," IEEE Signal Processing Magazine, vol. 25, no. 5, pp. 5780, 2008.

[7] Q. Liu, S. Zhou, and G. B. Giannakis, "Cross-layer combining of adaptive modulation and coding with truncated ARQ over wireless links," Wireless Communications, IEEE Transactions on, vol. 3, no. 5, pp. 1746-1755, 2004.

[8] _ - "Queuing with adaptive modulation and coding over wireless links: cross-layer analysis and design," Wireless Communications, IEEE Transactions on, vol. 4, no. 3, pp. 1142-1153, 2005.

[9] L. B. Le, E. Hossain, and A. S. Alfa, "Delay statistics and throughput performance for multi-rate wireless networks under multiuser diversity," IEEE Transactions on Wireless Communications, vol. 5, no. 11, pp. 3234-3243, 2006.

[10] M. M. Rashid, M. J. Hossain, E. Hossain, and V. K. Bhargava, "Opportunistic spectrum scheduling for multiuser cognitive radio: a queueing analysis," Wireless Communications, IEEE Transactions on, vol. 8, no. 10, pp. 5259-5269, 2009.

[11] J. Wang, A. Huang, L. Cai, and W. Wang, "On the queue dynamics of multiuser multichannel cognitive radio networks," Vehicular Technology, IEEE Transactions on, vol. 62, no. 3, pp. 1314-1328, 2013.

[12] S. Wang, B. T. Maharaj, and A. S. Alfa, "Resource allocation and performance measures in multi-user multi-channel cognitive radio networks," in Vehicular Technology Conference (VTC Spring), 2016 IEEE 83rd. IEEE, 2016, pp. 1-5.

[13] X. Gelabert, O. Sallent, J. Pérez-Romero, and R. Agustí, "Spectrum sharing in cognitive radio networks with imperfect sensing: a discretetime markov model," Computer Networks, vol. 54, no. 14, pp. 2519 2536, 2010.

[14] H.-J. Lim, D.-Y. Seol, and G.-H. Im, "Joint sensing adaptation and resource allocation for cognitive radio with imperfect sensing," IEEE Transactions on Communications, vol. 60, no. 4, pp. 1091-1100, 2012.

[15] A. Doufexi, S. Armour, M. Butler, A. Nix, D. Bull, J. McGeehan, and P. Karlsson, "A comparison of the HIPERLAN/2 and IEEE 802.11 a wireless LAN standards," Communications Magazine, IEEE, vol. 40, no. 5, pp. 172-180, 2002 .

[16] E. Malkamaki and H. Leib, "Performance of truncated type-ii hybrid ARQ schemes with noisy feedback over block fading channels," IEEE Transactions on Communications, vol. 48, no. 9, pp. 1477-1487, 2000.

[17] A. De Domenico, E. C. Strinati, and M.-G. Di Benedetto, "A survey on MAC strategies for cognitive radio networks," IEEE Communications Surveys \& Tutorials, vol. 14, no. 1, pp. 21-44, 2012.

[18] Q. Zhang, S. Kassam et al., "Finite-state Markov model for rayleigh fading channels," Communications, IEEE Transactions on, vol. 47, no. 11, pp. 1688-1692, 1999.

[19] G. Yu, Z. Zhang, and P. Qiu, "Efficient ARQ protocols for exploiting cooperative relaying in wireless sensor networks," Computer Communications, vol. 30, no. 14, pp. 2765-2773, 2007.

[20] T. G. Robertazzi, Computer networks and systems: queueing theory and performance evaluation. Springer Science \& Business Media, 2012.

[21] A. J. Goldsmith and S.-G. Chua, "Adaptive coded modulation for fading channels," Communications, IEEE Transactions on, vol. 46, no. 5, pp 595-602, 1998.

[22] H. S. Wang and N. Moayeri, "Finite-state Markov channel - a useful model for radio communication channels," Vehicular Technology, IEEE Transactions on, vol. 44, no. 1, pp. 163-171, 1995.

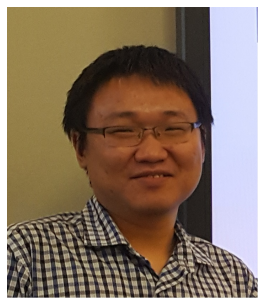

Shi Wang received B.Eng and M.Eng degree from Beijing University of Telecommunication, Beijing on 2006 and 2009, respectively. He is currently a $\mathrm{Ph} . \mathrm{D}$. student in the Department of Electrical, Electronic and Computer Engineering of the University of Pretoria, Pretoria, South Africa. His research focus includes cognitive radio networks, radio resource allocation in wireless networks and queueing theory.

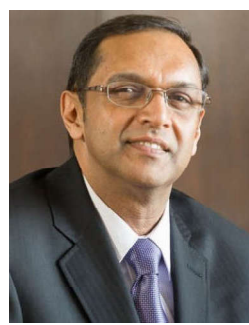

B. T. Maharaj Sunil Maharaj received his $\mathrm{PhD}$ in Engineering the area of Wireless Communications from the University of Pretoria. Dr Maharaj is a full professor and currently holds the research position of Sentech Chair in Broadband Wireless Multimedia Communications in the Department of Electrical, Electronic and Computer Engineering at the University of Pretoria. His research interests are in OFDM-MIMO systems, massive MIMO, cognitive radio resource allocation and 5G Cognitive Radio Sensor Networks.

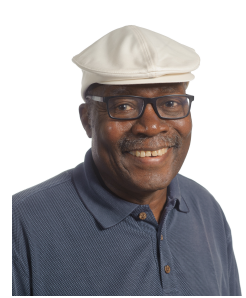

Attahiru S. Alfa is Professor Emeritus at the University of Manitoba, Department of Electrical and Computer Engineering and also a UP/CSIR cohosted SARChI Chair professor at the University of Pretoria, Department of Electrical, Electronic and Computer Engineering. His research covers, but not limited to, the following areas: queueing theory and applications, optimization, performance analysis and resource allocation in telecommunication systems, modeling of communication networks, analysis of cognitive radio networks, modeling and analysis of wireless sensor networks, developing efficient decoding algorithms for LDPC codes, channel modeling, traffic estimation for the Internet, and cross layer analysis. Dr. Alfa also works in the application of queueing theory to other areas such as transportation systems, manufacturing systems and healthcare systems. He has authored two books, "Queueing Theory for Telecommunications: Discrete Time Modelling of a Single Node System", published by Springer in 2010, and "Applied Discrete-Time Queue" published in 2015 also by Springer. 